\title{
Preparation of nano-hydroxyapatite/chitosan aqueous dispersions: From lab scale to continuous production using an innovative static mixer
}

\author{
G. Ruphuy ${ }^{\mathrm{a}, \mathrm{b}, \mathrm{c}}$, T. Weide ${ }^{\mathrm{a}}$, J.C.B. Lopes ${ }^{\mathrm{a}}$, M.M. Dias ${ }^{\mathrm{a}}$, M.F. Barreiro ${ }^{\mathrm{b}, \mathrm{d}, *}$ \\ a Laboratory of Separation and Reaction Engineering, Laboratory of Catalysis and Materials, Associate Laboratory LSRE-LCM, Faculty of Engineering, University of Porto, \\ Porto, Portugal \\ ${ }^{\mathrm{b}}$ Laboratory of Separation and Reaction Engineering, Laboratory of Catalysis and Materials, Associate Laboratory LSRE-LCM, Bragança Polytechnic Institute, Bragança, \\ Portugal \\ ${ }^{\mathrm{c}}$ Department of Chemical Engineering, University of Chemistry and Technology, Prague, Czech Republic \\ d Centro de Investigação de Montanha (CIMO), Instituto Politécnico de Bragança, Campus de Santa Apolónia, 5300-253 Bragança, Portugal
}

\section{A R T I C L E I N F O}

\section{Keywords:}

Chitosan

Nano-hydroxyapatite

Nano-composites

Dispersion stability

NETmix technology

\begin{abstract}
A B S T R A C T
Chitosan is widely used in the preparation of organic-inorganic composite materials, such as n-HAp/CS composites, which find application for bone regeneration. The methods for their preparation are various, and usually based on the preparation of intermediate $\mathrm{n}-\mathrm{HAp} / \mathrm{CS}$ dispersions, which can greatly influence the final properties of the resulting composites since it is expected that homogenous and stable dispersions lead to composite materials with improved final properties. This work hypothesizes that, additionally to process parameters such as $\mathrm{pH}, \mathrm{n}-\mathrm{HAp} / \mathrm{CS}$ weight ratio, mixing conditions and the presence of salts, chitosan itself has a high impact on dispersions stability. Thus, the importance of properly control the preparation of the n-HAp/CS intermediate dispersions is highlighted by doing a systematic study where relevant processing parameters were studied at lab scale using ultrasonication, alone or in the presence of chitosan, namely on particle size and zeta potential. Furthermore, and based on the best laboratorial conditions, the production of n-HAp/CS nanocomposite dispersions in continuous mode was attempted through NETmix ${ }^{\circledR}$ technology, an innovative static mixer and reactor developed at the Associate Laboratory LSRE-LCM of the Faculty of Engineering of the University of Porto (FEUP).
\end{abstract}

\section{Introduction}

Nanoparticles are extraordinary systems that, due to their high surface area-to-volume ratio, exhibit unique properties. Their incorporation into polymeric matrices, yet challenging, has been of great interest aiming at producing final materials with enhanced properties (Akindoyo, Beg, Ghazali, Heim, \& Feldmann, 2017; Pighinelli \& Kucharska, 2013; Zima, 2018). The preparation of such nanocomposites can benefit from the formulation of intermediate homogeneous dispersions, since it is expected that the large surface-to-volume ratio results in increased interfacial particle-matrix interactions, which can improve the overall properties of the resulting materials (Casarrubios et al., 2016; Šupová, Martynková, \& Barabaszová, 2011). However, the preparation of homogenous dispersions is still a complex task to accomplish. Intermolecular van der Waals forces between particles dominate when their sizes are below $100 \mathrm{~nm}$, causing their agglomeration or clustering. In addition, the difficulty in controlling the mixing between two dissimilar phases can lead to phase separation, resulting in products with limited properties (Kickelbick, 2007; Šupová et al., 2011).

The mineral phase of natural bone consists of blade-like crystals of nanometric size, very similar to stoichiometric hydroxyapatite nanoparticles, well distributed throughout collagen fibres. To mimic these natural materials, it is desirable to produce, as intermediates, highly homogeneous and stable n-HAp dispersions with close interaction with the organic component, to be further shaped into final structures that will serve as bone substitutes. Due to their interest for non-load-bearing bone graft applications, many methods have been reported to prepare n-HAp dispersions with chitosan as the organic component (Okada et al., 2017; Pangon, Saesoo, Saengkrit, Ruktanonchai, \& Intasanta, 2016; Shakir et al., 2018). One of the methods preferred by some authors is the so-called in situ co-precipitation (Chen, Wang, \& Lin, 2002; Danilchenko et al., 2011; Yamaguchi et al., 2001). In this method, the synthesis of HAp is carried out in the presence of chitosan in a neutral or basic environment ( $\mathrm{pH} \geq 7$ ), therefore, causing the precipitation of chitosan along with HAp simultaneously. Homogeneous incorporation

\footnotetext{
* Corresponding author at: Centro de Investigação de Montanha (CIMO), Instituto Politécnico de Bragança, Campus de Santa Apolónia, 5300-253 Bragança, Portugal.

E-mail address: barreiro@ipb.pt (M.F. Barreiro).
} 
of HAp into the chitosan matrix has been reported using this process, however, scaffolds produced from intermediate n-HAp/CS dispersions with $\mathrm{pH} \geq 7$ can lead to brittle structures with some impurities due to the presence of alkaline salts (Ruphuy et al., 2018).

An alternative attractive method consists in physically mixing previously synthesized HAp particles, usually in powder form or ultrasonically dispersed in water, into a chitosan solution. Typically, at labscale, this technique is carried out, by mixing both components in a vessel using a magnetic stirrer and/or ultrasonication, resulting in final hybrid materials with quite favorable physicochemical and biological properties (Atak et al., 2017; Kim et al., 2009; Nazeer, Yilgör, \& Yilgör, 2017; Thein-Han \& Misra, 2009; Zhao et al., 2002). This is a simple and straightforward technique, but it can lead to non-homogenous dispersions at a microscopic level due to the difficulty in controlling the mixing between the two dissimilar phases (Peniche, Solís, Davidenko, \& García, 2010; Šupová, 2009) and, therefore, originate final materials with weaker mechanical properties when compared to other methods (Chen et al., 2011; Hu, Li, Wang, \& Shen, 2004). In addition, this method implies the incorporation of HAp nanoparticles into an acidic environment, which might influence HAp solubility, phase stability and surface chemistry (Wilson \& Hull, 2008).

Despite the numerous publications on the preparation of HAp and $\mathrm{HAp} / \mathrm{CS}$ nanocomposite dispersions, studies regarding the influence of chitosan presence on HAp particles' surface properties are very scarce. Namely, Wilson et al. (2008) (Wilson \& Hull, 2008) was the only study found in which changes in HAp surface chemistry, colloidal stability and chemical composition were reported after aging nanophase HAp particles in chitosan acetate gel solutions for 30 days. Authors attributed the observed changes to solubility effects, considering that HAp becomes slowly soluble at $\mathrm{pH}$ below 5 , and the $\mathrm{pH}$ of the final HAp/CS mixtures was acidic ( $\mathrm{pH} 4-5)$. Lastly, it was also pointed out that chitosan not only adsorbs strongly to HAp particles, but also improves colloidal stability. Moreover, no studies were found regarding the effects of $\mathrm{pH}$ on particle size and zeta potential of $\mathrm{n}-\mathrm{HAp} / \mathrm{CS}$ dispersions. Studies were found regarding the colloidal stability of n-HAp in the presence of other components rather than chitosan, e.g. sodium citrate (Jin, Zhuang, Zhang, Guo, \& Tan, 2015) and sodium oleate (Vucinic, Radulovic, \& Deusic, 2010).

In this work, aqueous dispersions of nanohydroxyapatite (n-HAp), alone or in the presence of chitosan (CS), were prepared by ultrasonication. The produced dispersions were analyzed in what concerns particle size and zeta potential, then related with important parameters such as $\mathrm{pH}$, chitosan content, the presence of salts, and storage time. Furthermore, and having in view the interest to obtain these intermediate nanodispersions at pilot/industrial scale, a new method for the production of $\mathrm{n}$-HAp/CS nanocomposite dispersions in continuous mode is introduced using the NETmix technology, a novel static mixer and reactor (Lopes et al., 2005; Laranjeira, Martins, Lopes, \& Dias, 2009; Laranjeira, Martins, Nunes, Lopes, \& Dias, 2011). In fact, currently available methods for the preparation of n-HAp/CS dispersions are mainly based in batch processes. In this context, NETmix appears as a promising technology since it allows the production in continuous mode with high reproducibility and micromixing control. The NETmix reactor consists of a network of static mixing chambers interconnected by transport channels. Due to the organized configuration of unit cells, the total volume for actual mixing is restricted to the volume of the chambers since mixing between different streams occurs only in the chambers. Therefore, unlike other static mixers and conventional stirred tank reactors, micromixing can be controlled as it depends on the mixing behaviour of the chambers and channels; while the channels behave as plug-flow reactors, zones of total segregation, the chambers behave as perfectly mixed conventional stirred tank reactors, zones of complete micromixing (Laranjeira et al., 2009).

The micromixing quality is a key factor determining HAp properties, such as purity, particle size distribution, crystallinity and morphology and the NETmix reactor has been used to produce high quality nanocrystalline HAp (Gomes, Silva, Quadros, Dias, \& Lopes, 2009; Silva, Quadros, Laranjeira, Dias, \& Lopes, 2008); however, the use of NETmix for the production of nanocomposite dispersions has never been tested before, thus being introduced, for first time, in the present work.

\section{Materials and methods}

\subsection{Materials}

The nanodispersions were prepared using two different formulations of hydroxyapatite aqueous paste, both supplied by Fluidinova S.A.: nanoXIM-CarePaste composed of $15.5 \pm 0.5 \%$ wt. of HAp $\left(\mathrm{Ca}_{10}\left(\mathrm{PO}_{4}\right)_{6}(\mathrm{OH})_{2}\right.$ ) nanoparticles (particle size $<50 \mathrm{~nm}$ ), $4.5 \pm 0.5 \%$ wt. $\mathrm{KCl}$ and water content $\leq 81.0 \% \mathrm{wt}$; and nanoXIM-HAp102, composed of $15.0 \pm 1.0 \% \mathrm{wt}$. of HAp rod-like nanoparticles suspended in pure water (particle size $<50 \mathrm{~nm}$ ). Chitosan 90/200/A1 corresponding to flakes with size $<200 \mu \mathrm{m}$, deacetylation degree of $91.9 \%$, and dynamic viscosity of $128 \mathrm{mPa} \cdot \mathrm{s}\left(1 \%\right.$ at $20^{\circ} \mathrm{C}$ in $1 \%$ acetic acid solution) was acquired from Biolog-Heppe $\mathrm{GmbH}$ (Germany). Glacial acetic acid, sodium acetate tri-hydrate, and sodium hydroxide, all of analytical grade from Sigma Aldrich, were used to prepare the required solutions.

\subsubsection{Dispersions preparation at laboratorial scale}

Aqueous dispersions of nano-hydroxyapatite (n-HAp), alone or in the presence of chitosan (CS), were prepared at HAp concentrations of $1.6 \mathrm{~g} / \mathrm{l}, 6.4 \mathrm{~g} / \mathrm{l}$ and $20 \mathrm{~g} / \mathrm{l}$, corresponding to mean free distance between particles (calculated according to Eq. (1)) of 3.0, 1.5 and 0.70 respectively.

$\frac{\Delta x_{\text {part }}}{L_{\text {part }}}=\left(\frac{\pi}{4}\right)^{1 / 3}\left(\frac{\rho_{H A p}}{C_{H A p} \bullet M_{H A p}}\right)^{1 / 3}\left(\frac{D_{\text {part }}}{L_{\text {part }}}\right)^{2 / 3}-1$

where $\rho_{H A p}, C_{H A p}$, and $M_{H A p}$, are the density, concentration and molar mass of HAp respectively; and $L_{\text {part }}$ and $D_{\text {part }}$ are the length and diameter of one rod-like HAp nanoparticle, which was approximated to a cylindrical shape.

Samples were prepared by ultrasonication using a laboratorial scale Ultrasonic Processor (Sonicator) QSonica Q700, at 50\% amplitude with a maximum power output of $700 \mathrm{~W}$, frequency output of $20 \mathrm{kHz}$ and a $12.5 \mathrm{~mm}$ diameter horn with replaceable tip. Special care was taken to ensure that both, the geometry of the vessel and the position of the probe's tip, were fixed (see Fig. 1), since geometric factors affect the amount of energy absorbed by the particles due to their relative position. Samples were analyzed in what concerns the influence of ultrasonication time, the presence of salts (particularly $\mathrm{KCl}$ ), n-HAp/CS weight ratio, $\mathrm{pH}$, storage time, and HAp concentration on particle size and zeta potential.

2.1.1.1. Effect of ultrasonication time. The optimal ultrasonication parameters were determined by evaluating the influence of ultrasonication time on the particle size of the n-HAp aqueous dispersions. Dispersions of $\mathrm{n}-\mathrm{HAp}$ alone and in the presence of chitosan were prepared $\left(\mathrm{C}_{\mathrm{HAp}}=1.6 \mathrm{~g} / \mathrm{l}, 6.4 \mathrm{~g} / \mathrm{l}\right.$ and $\left.20 \mathrm{~g} / \mathrm{l}\right)$ and subjected to different ultrasonication time periods, ranging from 0 to $100 \mathrm{~s}$. Emphasis was given to the production of n-HAp/CS dispersions with a weight ratio of $70 / 30$ since it represents the typical bone composition (Burr \& Allen, 2013). To avoid the precipitation of chitosan, as well as the solubilization of the HAp, the $\mathrm{pH}$ of all prepared n-HAp/CS samples was checked to be of $\mathrm{pH} 5.5$ at room temperature. Particle size of the samples was examined by dynamic light scattering (DLS). The optimal parameters were identified and used for the preparation of the nanodispersions used in further studies (presence of salts, $\mathrm{n}-\mathrm{HAp} / \mathrm{CS}$ weight ratio, $\mathrm{pH}$, storage time and HAp concentration). 


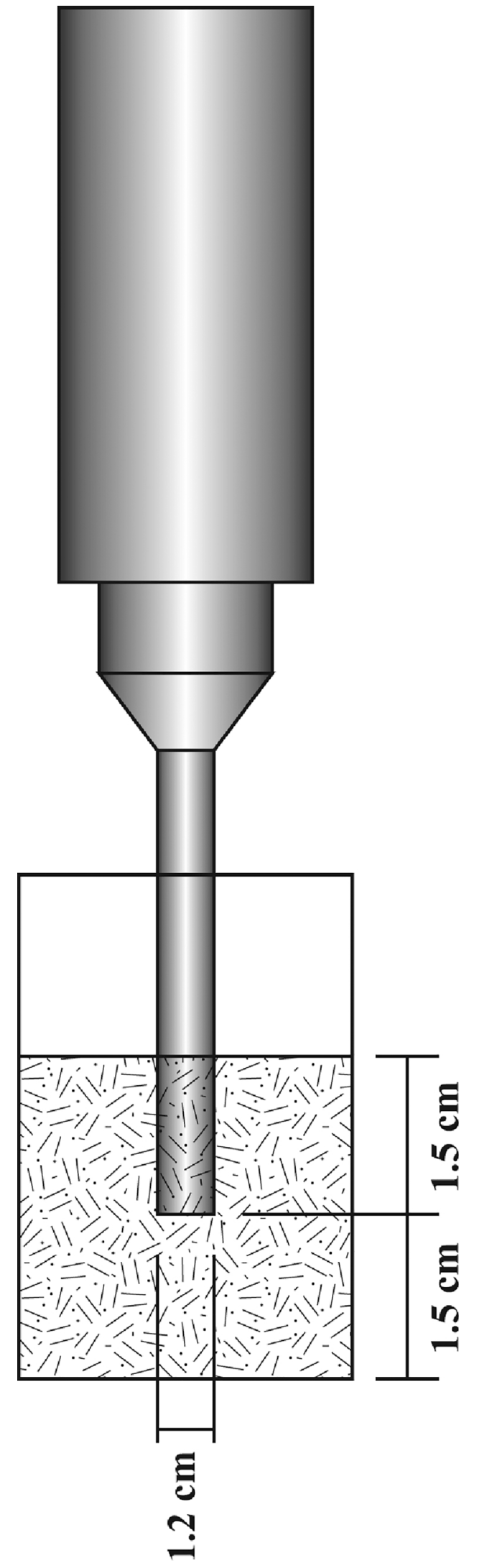

Fig. 1. Experimental set-up used at laboratorial scale.

2.1.1.2. Effect of the presence of salts. Given that one of the hydroxyapatite pastes used for the preparation of the nanodispersions, nanoXIM-CarePaste, contains $\mathrm{KCl}$ in its formulation, the influence of this salt on particle size and zeta potential was evaluated by DLS and electrophoretic light scattering (ELS) respectively. For that, $\mathrm{n}-\mathrm{HAp}$ dispersions $\left(\mathrm{C}_{\mathrm{HAp}}=1.6 \mathrm{~g} / \mathrm{l}\right)$, both alone and in the presence of chitosan (n-HAp/CS weight ratio $=70 / 30$ ), were prepared using the nanoXIM-CarePaste, after being washed three times with deionized water. In parallel, dispersions containing nanoXIMCarePaste with $\mathrm{KCl}$, as originally provided, were also prepared and characterized.

2.1.1.3. Effect of $n-H A p / C S$ weight ratio. The influence of the presence of chitosan was also studied. For that, nanodispersions $\left(\mathrm{C}_{\mathrm{HAp}}=1.6 \mathrm{~g} / \mathrm{l}\right)$ with different n-HAp/CS weight ratios were prepared and analysed by DLS and ELS. Emphasis was given to n-HAp as the main component, therefore, the nanodispersions were prepared considering n-HAp/CS weight ratios of 100/0, 90/10, 80/20, 70/30, 60/40, and 50/50.

2.1.1.4. Influence of $p H$ on the electrostatic stability of the nanodispersions. Titration of n-HAp nanodispersions $\left(\mathrm{C}_{\mathrm{HAp}}=1.6 \mathrm{~g} / \mathrm{l}\right)$, alone and in the presence of chitosan (n-HAp/CS weight ratio $=70$ / 30), were carried out using $\mathrm{CH}_{3} \mathrm{COOH} 0.20 \mathrm{M}$ and $\mathrm{NaOH} 0.25 \mathrm{M}$ as titrants. Changes in electrostatic stability (zeta potential) due to $\mathrm{pH}$ variations were evaluated.

2.1.1.5. Effect of storage time. The electrostatic stability of n-HAp/CS dispersions $\left(\mathrm{C}_{\mathrm{HAp}}=1.6 \mathrm{~g} / \mathrm{l}\right.$ and $\mathrm{n}-\mathrm{HAp} / \mathrm{CS}$ weight ratio $\left.=70 / 30\right)$ along time was studied. The nanodispersion was prepared by ultrasonication using the optimized process conditions, and stored in a refrigerator at $4{ }^{\circ} \mathrm{C}$. The zeta potential was measured at times ranging from 0 (right after ultrasonication) to 30 days.

2.1.1.6. Characterization of $n$-HAp aqueous dispersions with higher concentration. n-HAp/CS dispersions with concentration $\mathrm{C}_{\mathrm{HAp}}=20 \mathrm{~g} / 1$ $(\mathrm{pH} 5.5$, without $\mathrm{KCl}$ and $\mathrm{n}-\mathrm{HAp} / \mathrm{CS}$ weight ratio $=70 / 30)$ were prepared and, since it was determined that such dispersion concentration was unsuitable for DLS, particle size distributions (PDS) in volume were obtained by laser diffraction, both for n-HAp dispersions alone and in the presence of chitosan. The main objective of this measurement was to infer the impact of chitosan on particle's size and stability.

\subsubsection{Dispersion preparation in continuous mode}

n-HAp/CS nanocomposite dispersions were prepared by a continuous process using the best formulation according to the results achieved at lab scale. For that, hydroxyapatite nanoparticles were incorporated in the chitosan solution, with rigorous control of $\mathrm{pH}$ conditions, using a NETmix reactor (see Fig. 2a). A "solution A" was prepared consisting of $3 \%$ chitosan solution in $1 \%$ acetic acid prepared with acetate buffer ( $\mathrm{pH} 5.5$ at room temperature) till reaching the desired concentration. A "solution B", consisting of nanoXIM-HAp102 aqueous paste (without $\mathrm{KCl}$ ) and distilled water $(\mathrm{pH} 8.5$ at room temperature) in adequate proportions, was also prepared. The concentrations of solutions A and B were set such that the n-HAp/CS final dispersions had n-HAp concentration of $20 \mathrm{~g} / \mathrm{l}$ and a n-HAp/CS weight ratio of $70 / 30$, the typical bone composition. The produced n-HAp/CS hybrid dispersions had a final $\mathrm{pH}$ value of approximately 5.5 (room temperature).

As a first approach, solutions A and B were injected in the reactor using the configuration shown in Fig. $2 \mathbf{b}$. The quantities of chitosan and nanoXIM-HAp102 aqueous paste used to prepare the respective solutions are presented in Table 1 . The test was carried out twice using the same conditions. Results are reported as "experiment 1 " and "experiment 2".

\subsubsection{Characterization}

Particle size and zeta potential of the prepared nanodispersions $\left(\mathrm{C}_{\mathrm{HAp}}=1.6 \mathrm{~g} / \mathrm{l}\right.$ and $\left.6.4 \mathrm{~g} / \mathrm{l}\right)$ were determined by dynamic and 


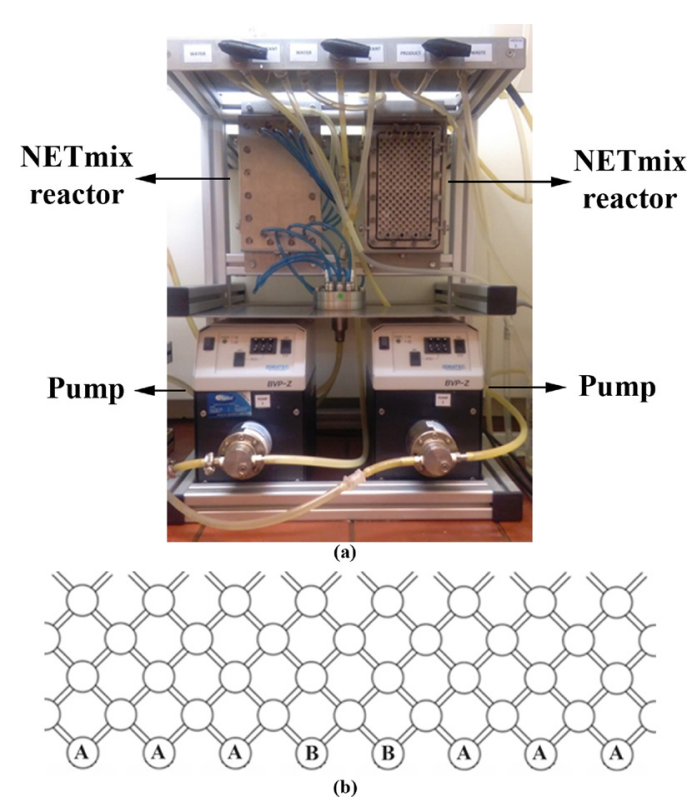

Fig. 2. (a) NETmix reactor: Experimental set-up used for the dispersion preparation in continuous mode; (b) Injection scheme used for the preparation of n-HAp/CS hybrid dispersions: solution A (A) and solution B (B).

Table 1

Quantities of chitosan and nanoXIM-HAp102 aqueous paste used to prepare the solutions A and B to be injected in the NETmix reactor.

\begin{tabular}{lll}
\hline Component & Solution A & Solution B \\
\hline Volume of chitosan 3\% in acetic acid 1\% (ml) & 289 & 0 \\
Volume of acetate buffer, pH 5.5 (ml) & 511 & 0 \\
Volume of nanoXIM-HAp102 aqueous paste 15\% $(\mathrm{ml})$ & 0 & 135 \\
Volume of distilled water (ml) & 0 & 65 \\
Total volume $(\mathrm{ml})$ & 800 & 200 \\
\hline
\end{tabular}

electrophoretic light scattering, respectively, using a Zetasizer Nano ZS from Malvern Instruments; model ZEN 3600 with a 633-nm red laser. Temperature and $\mathrm{pH}$ were also monitored during the preparation process. The effect of $\mathrm{pH}$ on the zeta potential was studied by performing titrations using the MPT-2 Autotitrator, an external device connected to the Zetasizer Nano ZS from Malvern Instruments. Moreover, dispersions with higher n-HAp concentration $\left(\mathrm{C}_{\mathrm{HAp}}=20 \mathrm{~g} / \mathrm{l}\right)$, alone and in the presence of chitosan, were characterized by laser diffraction spectroscopy using a Coulter LS-230.

\subsection{Results and discussion}

\subsubsection{Effect of ultrasonication time}

Ultrasonication is a common laboratory method used either for reduction of particle size or agglomeration disruption. Bearing in mind that a large amount of energy is released due to the collapse of thousands of microscopic bubbles, causing clusters disruption, energy efficiency was firstly considered in the present study. The optimal process parameters were determined by running experiments at fixed amplitude $(50 \%)$ and by varying the ultrasonication time. Fig. 3 shows the results obtained relative to the changes in particle size for different pre-defined ultrasonication times, as well as its variation with energy input. A logarithmic tendency of particle size to decrease with increasing ultrasonication time was observed for all analyzed samples (dispersions of HAp alone and n-HAp/CS dispersions). Furthermore, it was observed that, for relatively low energy input (processing times of 10-30 s), large agglomerates were completely broken down into smaller ones; however, larger particle sizes were detected in the case of the n-HAp/CS dispersions, suggesting that some HAp nanoparticles remain as clusters
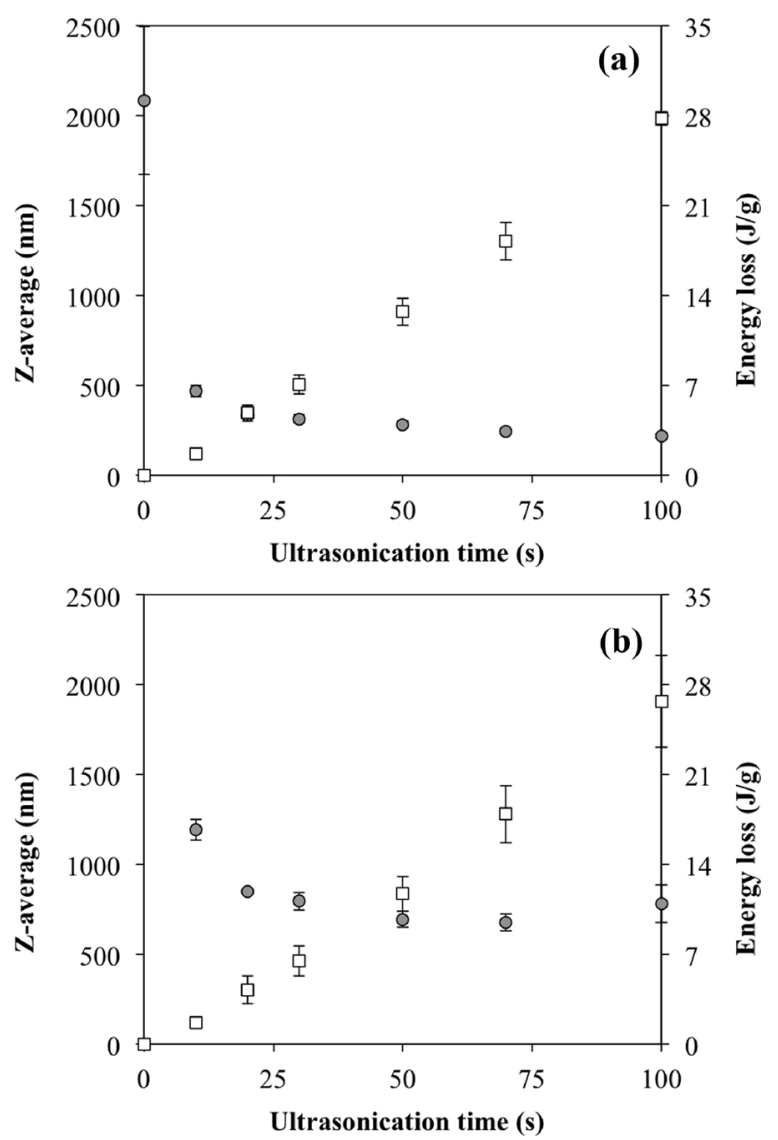

Fig. 3. Z-average (filled circle) and energy loss (white square) versus ultrasonication time of $\mathrm{n}-\mathrm{HAp} / \mathrm{CS}$ dispersions of concentrations: (a) $1.6 \mathrm{~g} / \mathrm{l}$; (b) $6.4 \mathrm{~g} / \mathrm{l}$. All measurements show SD based on 3 replicas.

when dispersed in chitosan solutions.

Relative to zeta potential, data exhibited low variability for all the examined samples, with values comprised between +27 and $+30 \mathrm{mV}$ $(9.0<\mathrm{pH}<10.0)$ for dispersions of HAp alone, and higher values, ranging between +32 to $+34 \mathrm{mV}$ ( $\mathrm{pH} 5.5$ ), for the n-HAp/CS dispersions. These results suggest that, even when larger particle sizes are obtained by particle agglomeration in the presence of chitosan, the nHAp/CS dispersions exhibit better electrostatic stability. Generally, if a dispersion is not stabilized, by electrostatic, steric, or electrosteric effects, nanoparticles tend to cluster again shortly after ultrasonication. Stabilization through electrosteric effects can be achieved by addition of a polyelectrolyte (Nguyen, Rouxel, Hadji, Vincent, \& Fort, 2011). Therefore, the observed increase of zeta potential observed in the nHAp/CS dispersions, relative to dispersions of n-HAp alone, may be due to electrosteric effects imparted by chitosan.

In addition, the results obtained from the energy balance for both, n-HAp alone and n-HAp/CS samples, within the studied HAp range of concentrations ( $1.6 \mathrm{~g} / \mathrm{l}, 6.4 \mathrm{~g} / \mathrm{l}$ and $20 \mathrm{~g} / \mathrm{l})$, point out that energy loss as heat increases linearly with ultrasonication time, reaching values from 25 to $30 \mathrm{~J} / \mathrm{g}$ at $100 \mathrm{~s}$ of ultrasonication, the higher time tested. Considering this and the tendency of particle size to decrease with increasing ultrasonication times, an energy input of approximately $50 \mathrm{~J} / \mathrm{g}$ ( $30 \%$ of energy loss), corresponding to $50 \mathrm{~s}$ of ultrasonication time at $50 \%$ amplitude, is considered optimal since higher ultrasonication times do not significantly affect HAp particle size.

Dispersions with the lowest concentration $\left(\mathrm{C}_{\mathrm{HAp}}=1.6 \mathrm{~g} / \mathrm{l}\right)$ are considered more suitable for DLS measurements. Therefore, the study of the effects of the presence of salts (particularly $\mathrm{KCl}$ ), n-HAp/CS weight ratio, $\mathrm{pH}$, and storage time were carried out for nanodispersions with this concentration and subjected to an ultrasonication time of $50 \mathrm{~s}(50 \%$ 

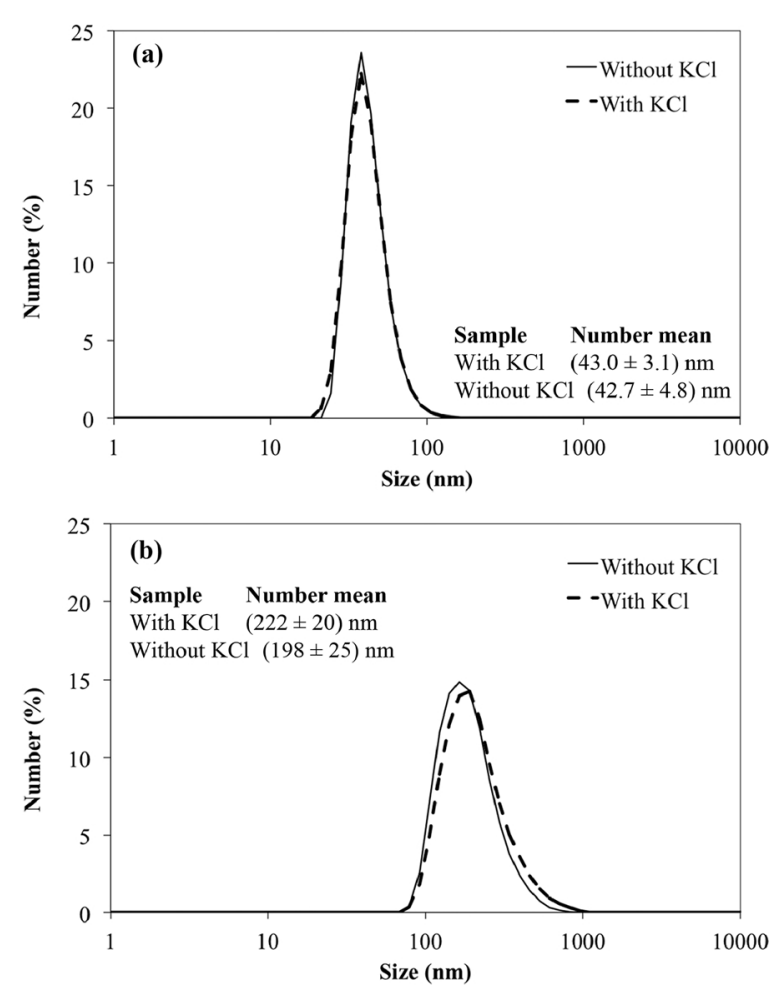

Fig. 4. Particle size distributions in number for dispersions prepared with washed nanoXIM-CarePaste (without $\mathrm{KCl}$ ) and with nanoXIM-CarePaste as originally provided (with $\mathrm{KCl}$ ): (a) n-HAp alone and (b) n-HAp/CS.

amplitude), corresponding to the optimal energy input.

\subsubsection{Effect of presence of salts}

The presence of salts affects the ionic strength of aqueous media. In particular, when salts are present in the former nanocomposite aqueous dispersions, the ionic content influences both, the surface charge of the nanoparticles and the charge of certain polysaccharides (e.g. chitosan), and therefore the polymer chain conformation (Carneiro-da-Cunha, Cerqueira, Souza, Teixeira, \& Vicente, 2011). Consequently, the nanoparticle-polymer interfacial interactions can change, influencing the overall properties of the produced nanocomposite material.

Particle size distributions (PDS) in number obtained for n-HAp dispersions alone and in the presence of chitosan, with and without $\mathrm{KCl}$, are shown in Fig. 4. Monodispersed distributions were obtained in all cases, and no considerable differences were observed in terms of size due to the presence of $\mathrm{KCl}$. Therefore, particle size increase is caused by the presence of chitosan and no evidence was found that $\mathrm{KCl}$ has an effect on the particle size of n-HAp dispersions.

While no important effect was observed in particle size due to the presence of $\mathrm{KCl}$, its presence influences the electrostatic stability of the dispersions, as shown in a previously published work of the group (Ruphuy, Saralegi, Lopes, Dias, \& Barreiro, 2016). In fact, the HAp nanoparticles dispersed in water, in the presence of $\mathrm{KCl}$, showed an improved stability, whereas for the hybrid n-HAp/CS dispersions, the opposite was observed. In the case of n-HAp/CS dispersions with $\mathrm{KCl}$, a good zeta potential value $(+32.8 \pm 0.2 \mathrm{mV})$ was obtained as well, but lower when compared with $\mathrm{n}-\mathrm{HAp} / \mathrm{CS}$ without $\mathrm{KCl}(+33.7 \pm 0.2 \mathrm{mV})$, indicating that the stabilizing effect of chitosan is better without the interference of salts. The differences observed could be attributed to different interactions between salt ions with the n-HAp particles surface and with chitosan chains, which carry a positive charge in solution.

\subsubsection{Effect of n-HAp/CS weight ratio}

As discussed before, larger particle sizes are obtained when HAp
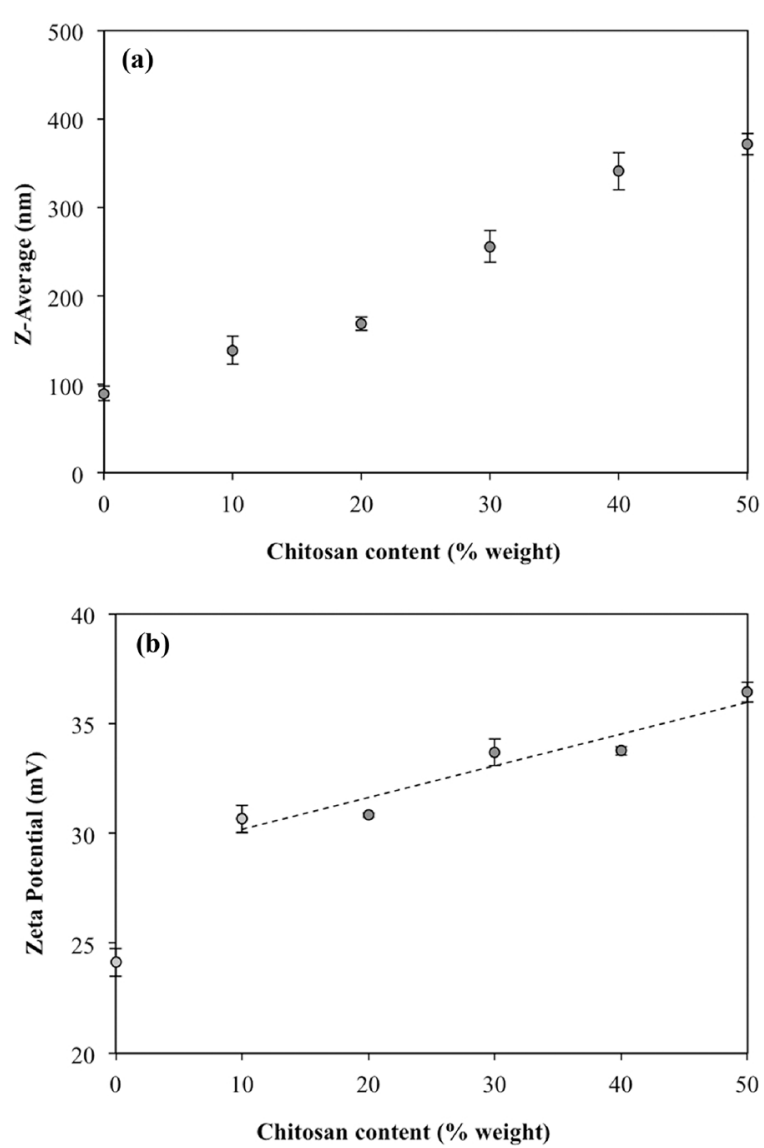

Fig. 5. Effect of chitosan content on n-HAp dispersions on: (a) Particle size; (b) Zeta Potential.

nanoparticles are dispersed in the presence of chitosan. Moreover, a linear tendency of particle size to increase with chitosan content was obtained (Fig. 5). In terms of zeta potential, chitosan incorporation into the $\mathrm{n}$-HAp dispersions leads to values above $+30 \mathrm{mV}$, showing a linear tendency to increase with chitosan content as well. Summarizing, the results show that even with the lower chitosan contents, the stability of the nanodispersions improves considerably due to the presence of chitosan.

The observed effect can be justified by the generated chemical interactions between HAp and chitosan, which include interactions between HAp calcium ions and chitosan amino groups $\left(\mathrm{NH}_{2}\right)$ (formation of coordination bonds), between HAp hydroxyl groups (OH) and chitosan $\mathrm{NH}_{2}$ or $\mathrm{OH}$ groups (formation of hydrogen bonds) (Venkatesan \& Kim, 2010; Xianmiao et al., 2009), and HAp phosphate groups $\left(\mathrm{H}_{2} \mathrm{PO}_{4}{ }^{-}\right)$and chitosan amino groups when positively charged $\left(\mathrm{NH}_{3}{ }^{+}\right)$ (Fadeeva, Barinov, Fedotov, \& Komlev, 2011). The last interactions described are the most probable to occur at $\mathrm{pH}$ 5.5.

In addition, being a polyelectrolyte, chitosan can cause an electrosteric stabilizing effect in the nanodispersions, i.e. the stabilization might be caused by both, steric and electrostatic mechanisms. Upon added to the n-HAp nanodispersions, chitosan can form a coating around the particles leading to stabilization by steric repulsions. As the chitosan content increases the coating gets thicker, which may also influence the observed increase in particle size. At the same time, charge stabilization takes place due to chitosan positive charges, which causes a linear increase in zeta potential as chitosan content increases. Therefore, even when the presence of chitosan in n-HAp dispersions increases particle size by particle aggregation, it improves dispersion stability, which is a desirable characteristic. 

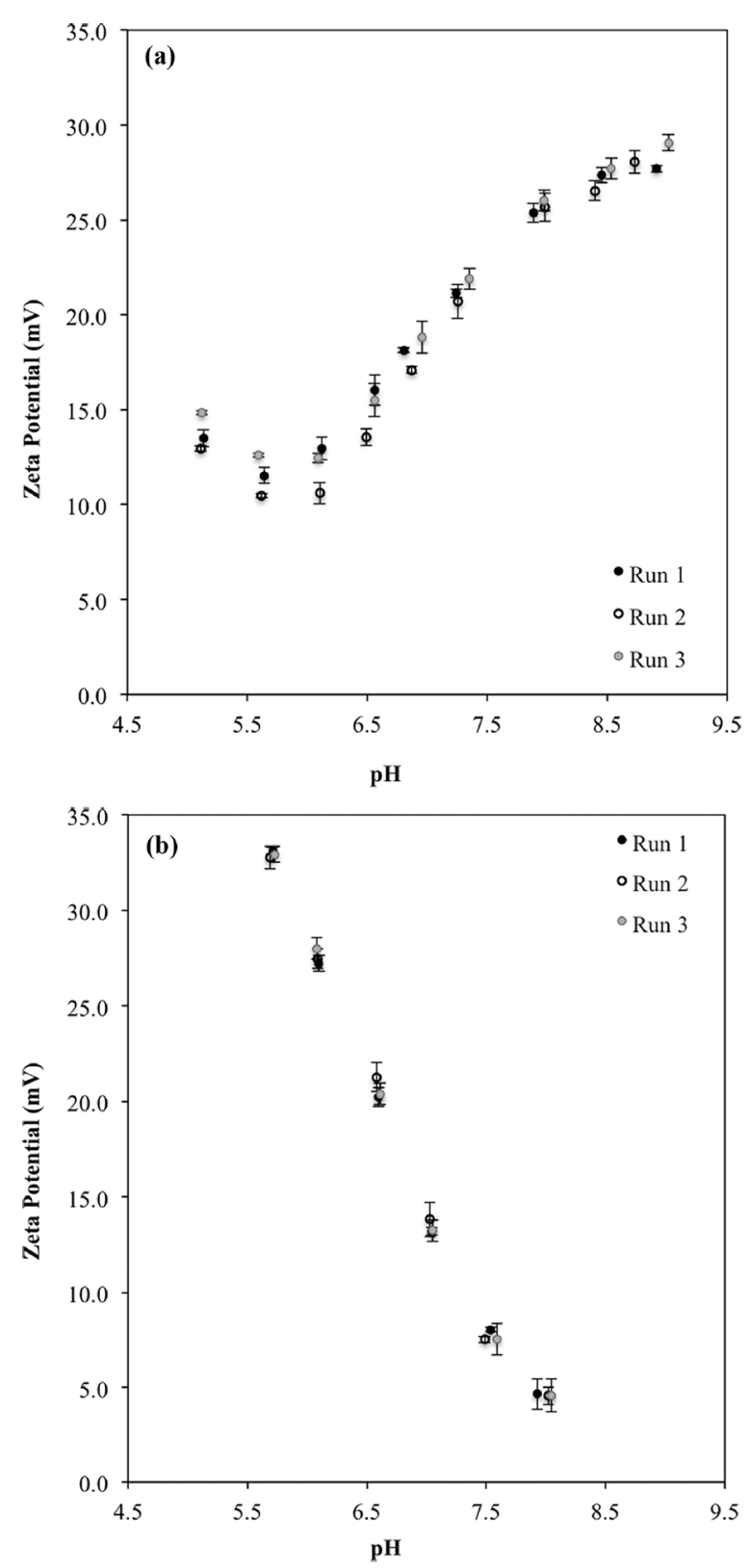

Fig. 6. Zeta potential dependency on $\mathrm{pH}$ for aqueous nanodispersions of: (a) nHAp dispersions alone using $\mathrm{CH}_{3} \mathrm{COOH} 0.20 \mathrm{M}$ (1 wt.\%) as titrant; and (b) nHAp/CS dispersions using $\mathrm{NaOH} 0.25 \mathrm{M}$ as titrant.

\subsubsection{Influence of $p H$ on the electrostatic stability of the nanodispersions}

The surface charge of particles dispersed in aqueous media depends upon the nature of the particle and the surrounding medium. In this context, ionization of surface groups and adsorption of charged particles are the two most important mechanisms defining surface charge of colloidal particles.

In the case of ionic solids, such as hydroxyapatite, the origin of surface charge is somewhat complex. The involved mechanism consists in differential ion dissolution from a crystal lattice (Prakash, Mishra, Malviya, \& Sharma, 2014), i.e. the net charge of n-HAp particles in aqueous medium arises from the unequal dissolution of surface ions and may result in either a net positive or a net negative surface charge. This mechanism is, as one would expect, highly dependent on the medium $\mathrm{pH}$. The development of the particle surface net charge affects the ions distribution in the surrounding interfacial region and, consequently, the zeta potential.

Fig. 6 shows the results obtained from the autotitrations of dispersions of n-HAp alone (in water) and in the presence of chitosan. In alkaline medium ( $\mathrm{pH} 9.0$ ), dispersions of n-HAp alone exhibit good electrostatic stability, with a zeta potential value of around $+29 \mathrm{mV}$. However, as the $\mathrm{pH}$ decreases, a tendency of zeta potential to decrease is observed, as shown in Fig. 6a. This tendency can be attributed to the $\mathrm{pH}$-dependent ion dissolution mechanisms, and resulting surface charge modification.

Due to their typical rod-like morphology, fact that favors the presence of calcium ions $\left(\mathrm{Ca}^{2+}\right)$ on the surface, HAp particle's net charge is shifted positive (Uskoković \& Uskoković, 2011). At high pH values, the HAp nanoparticles have a rather positive surface net charge with phosphate groups in the form of $\mathrm{PO}_{4}{ }^{3-}$ strongly bonded to the $\mathrm{Ca}^{2+}$ ions aligned on the surface. As $\mathrm{pH}$ decreases to $4<\mathrm{pH}<6$, unequal dissolution of HAp surface ions occurs; particularly, HAp loses $\mathrm{Ca}^{2+}$ ions to acetic acid to form calcium acetate, and the balance of HAp phosphate species is slowly shifted towards the formation of $\mathrm{H}_{2} \mathrm{PO}_{4}{ }^{-}$, causing the surface net charge to become less positively charged. Consequently, in slightly acidic environment ( $\mathrm{pH}$ 5.5) the electrostatic stability of n-HAp particles dispersed in water is poor, with zeta potential between +10 and $+15 \mathrm{mV}$.

In the case of the nanodispersions containing chitosan (Fig. 6b), a high zeta potential $(>+30 \mathrm{mV})$ is obtained at low $\mathrm{pH}$ values $(\mathrm{pH}<$ 6.5) and, oppositely to what happens with the dispersions of n-HAp alone, a linear tendency of zeta potential to increase with the decrease of $\mathrm{pH}$ is observed. This behavior can be explained by the adsorption mechanism of charged species. At $4<\mathrm{pH}<6$, formation of $\mathrm{H}_{2} \mathrm{PO}_{4}{ }^{-}$ is favored on HAp, while chitosan acquires positive charges $\left(\mathrm{NH}_{2} \rightarrow\right.$ $\mathrm{NH}_{3}{ }^{+}$), promoting the adsorption of chitosan onto HAp particle's surface, leading to a rather positively charged surface. Accordingly, at low $\mathrm{pH}$ values $(\mathrm{pH}<6.5)$ the presence of chitosan significantly improves the stability of n-HAp dispersions.

\subsubsection{Effect of storage time}

The electrostatic stability of n-HAp/CS dispersions was further studied by measuring zeta potential along time. The analysed dispersion, with $\mathrm{C}_{\mathrm{HAp}}=1.6 \mathrm{~g} / \mathrm{l}$ and $\mathrm{pH} \sim 5.5$, was prepared using the optimized process conditions, ultrasonication time of $50 \mathrm{~s}$ (50\% amplitude), and store in a refrigerator at $4{ }^{\circ} \mathrm{C}$. The zeta potential was measured at times ranging from 0 , right after ultrasonication, to 30 days. Results in Fig. 7 show that the zeta potential values were fairly constant along the time period analyzed, ranging between +36 and $+42 \mathrm{mV}$. This suggests that the prepared n-HAp/CS dispersions exhibit good electrostatic stability even for long time periods.

\subsubsection{Aqueous n-HAp dispersions with higher concentration}

Previously, diluted dispersions of n-HAp, with $\mathrm{C}_{\mathrm{HAp}}=1.6 \mathrm{~g} / \mathrm{l}$, were prepared and characterised to study the influence of different parameters on particle size and zeta potential. However, properties of

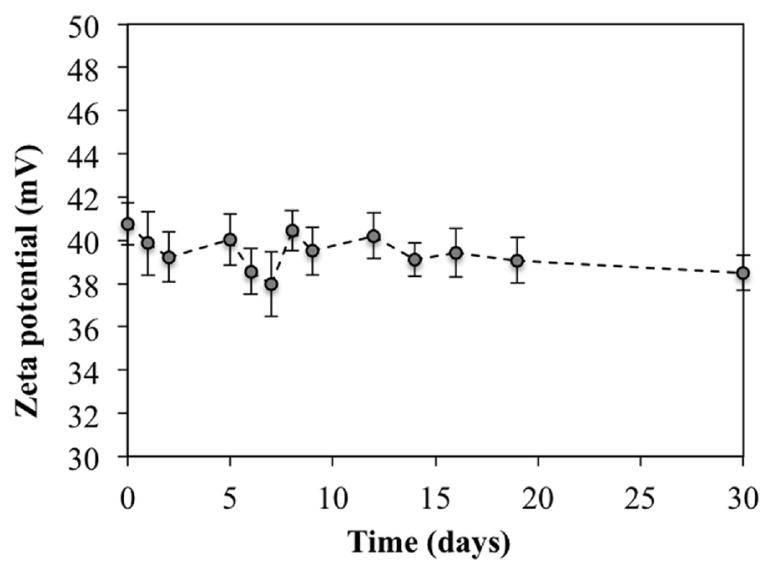

Fig. 7. Effect of storage time on zeta potential. 

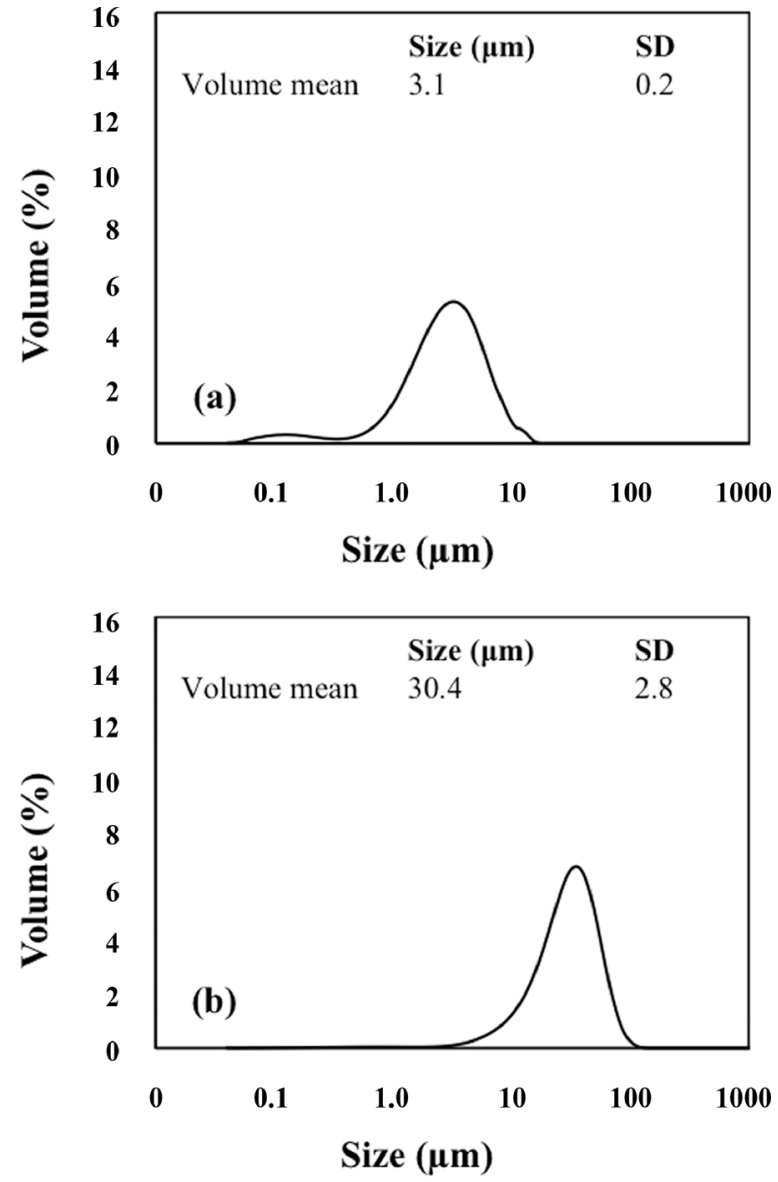

Fig. 8. Particle size distributions in volume by laser diffraction for n-HAp dispersions with concentrations of $20 \mathrm{~g} / \mathrm{l}$ : (a) n-HAp alone; (b) n-HAp/CS dispersions.

colloidal systems may change as a function of concentration. Having in view the potential commercialization of $\mathrm{n}-\mathrm{HAp} / \mathrm{CS}$ products, the production of hybrid dispersions with concentrations higher than $\mathrm{C}_{\mathrm{HAp}}=1.6 \mathrm{~g} / \mathrm{l}$ is desirable. Therefore, $\mathrm{n}$-HAp/CS dispersions with concentration $\mathrm{C}_{\mathrm{HAp}}=20 \mathrm{~g} / \mathrm{l}$ were prepared and, since it was determined that such dispersions were unsuitable for DLS, particle size distributions (PDS) in volume were obtained by laser diffraction, both for n-HAp dispersions alone and in the presence of chitosan. Results obtained are shown in Fig. 8. It is important to highlight that the

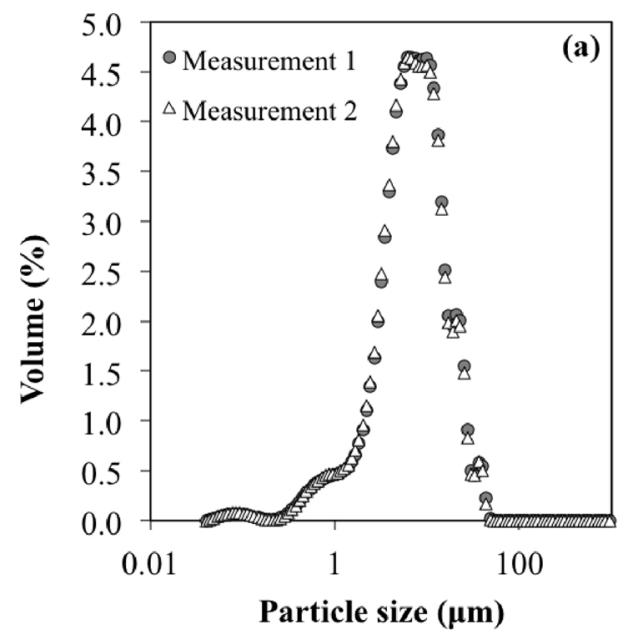

obtained results in this section are not intended for comparison with previous results obtained by DLS, but only to evaluate particle size of nHAp dispersions at higher concentrations.

It is observed that HAp nanoparticles form aggregates at this concentration level, $\mathrm{C}_{\mathrm{HAp}}=20 \mathrm{~g} / \mathrm{l}$, both for $\mathrm{n}$-HAp dispersions alone and in the presence of chitosan. Furthermore, the agglomerates formed are larger in the presence of chitosan, in accordance with results previously discussed. Aggregation is caused by the collision and subsequent adhesion of particles. Collision of particles is mainly due to their Brownian motion, and thus, mainly governed by the flow regime and the effects of external fields. In addition, particle collisions are affected by the double layer interactions, which determine the repulsive forces. Finally, particle concentration is the third factor affecting particle aggregation since collisions frequency increases with particle concentration (Babick, 2016). Therefore, the increase in particle size obtained for both types of $\mathrm{n}$-HAp dispersions can be attributed to the increase in particles collision frequency, and the differences observed due to the incorporation of chitosan are probably related mainly to differences in the flow regime. However, even at high concentrations, n-HAp dispersions in the presence of chitosan showed improved electrostatic stability with zeta potential values $>+30 \mathrm{mV}(\mathrm{pH} \sim 5.5)$.

\subsubsection{Production of $n-H A p / C S$ dispersions in continuous using NETmix technology}

Based on the results achieved for batch mode, the most adequate formulation for production in continuous consists of: n-HAp/CS nanodispersions with higher HAp concentration $\left(\mathrm{C}_{\mathrm{HAp}}=20 \mathrm{~g} / \mathrm{l}\right)$, which is more adequate for the production of final products with potential for commercialization, namely microparticles and scaffolds (Ruphuy et al., 2016, 2018) ; n-HAp/CS weight ratio of 70/30, which mimics bone composition and also exhibits good electrostatic stability; $\mathrm{pH}$ 5.5, which also presents better electrostatic stability than higher $\mathrm{pH}$ values; and finally, based on $\mathrm{n}-\mathrm{HAp}$ paste without $\mathrm{KCl}$, since its presence does not improve zeta potential of the composite dispersions, and may even compromise it.

Hydroxyapatite nanoparticles were successfully incorporated into the chitosan solution in continuous using the NETmix reactor at flow rates corresponding to low Reynolds number $(\operatorname{Re}=70)$. The low Reynolds number is due to the high viscosity of solution A (chitosan), which requires of high pump velocities; thus, with a $\mathrm{Re}=70$ the pump velocity is near the maximum capacity of the pump. The produced nHAp/CS hybrid dispersions were highly homogeneous and stable, with particle sizes in the order of $10 \mu \mathrm{m}$ and zeta potential $>+30 \mathrm{mV}(\mathrm{pH}$ 5.5 , room temperature).

The size distributions in volume are presented in Fig. 9, showing that the particle size distributions obtained are not only comparable to

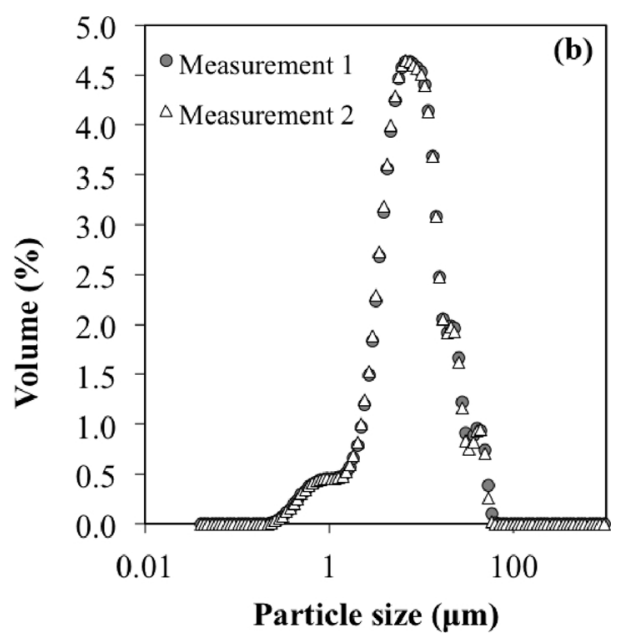

Fig. 9. Particle size distributions in volume obtained for the n-HAp/CS hybrid dispersions obtained using Netmix reactor: (a) experiment 1 ; (b) experiment 2. 
the ones obtained for the analogous samples prepared by ultrasonication (Fig. 8b) but also that the experiments were highly reproducible. The process can be further optimized but the obtained results are promising and prove the viability of using the NETmix static mixer for the production in continuous of $\mathrm{n}-\mathrm{HAp} / \mathrm{CS}$ nanocomposite dispersions.

\subsection{Conclusions}

Aqueous dispersions of n-HAp alone and in the presence of chitosan were prepared at laboratorial scale by ultrasonication and new insights on charge stability and particle size were investigated. It was found that $\mathrm{pH}$ greatly influences the zeta potential of n-HAp dispersions. In slightly acidic environment ( $\mathrm{pH}$ 5.5), the presence of chitosan greatly improves the electrostatic stability of n-HAp dispersions, exhibiting zeta potential values $>+30 \mathrm{mV}$. Furthermore, measurements carried out at different $\mathrm{n}$-HAp/CS weight ratios indicated that the zeta potential increases linearly with chitosan content increase. The presence of salts was also analysed, showing that the stabilizing effect of chitosan for the n-HAp dispersions is more effective without the interference of salts.

Finally, based on the best conditions achieved at lab-scale by ultrasonication, n-HAp/CS composite dispersions were produced in continuous mode using the NETmix reactor. It was proven that even at low Reynolds numbers, highly homogeneous and stable n-HAp/CS composite dispersions were produced. The produced dispersions presented particle sizes in the order of $10 \mu \mathrm{m}$ and zeta potential values $>+30$ $\mathrm{mV}$. The process exhibited excellent reproducibility and, even it can be further optimized, the obtained results proved the viability of NETmix static mixer to produce, in continuous mode, n-HAp/CS nanocomposite dispersions with desirable properties, process that can be easily scaledup, having in view a possible industrial scale.

\section{Acknowledgements}

Financial support for this work was provided in part by project Project POCI-01-0145-FEDER-006984 - Associate Laboratory LSRELCM funded by FEDER through COMPETE2020 - Programa Operacional Competitividade e Internacionalização (POCI) - and by national funds through FCT - Fundação para a Ciência e a Tecnologia, and by “AIProcMat@N2020 - Advanced Industrial Processes and Materials for a Sustainable Northern Region of Portugal 2020", with reference NORTE-01-0145-FEDER-000006, supported by NORTE 2020 under the Portugal 2020 Partnership Agreement, through the European Regional Development Fund (ERDF). FCT and FEDER under Programme PT2020 for financial support to CIMO (UID/AGR/00690/2013). G. Ruphuy thanks Universidad de Costa Rica (UCR) and Ministerio de Ciencia, Tecnología y Telecomunicaciones de Costa Rica (MICITT) for her scholarship. Authors thank Fluidinova S.A. for providing the HAp samples.

\section{References}

Akindoyo, J. O., Beg, M. D. H., Ghazali, S., Heim, H. P., \& Feldmann, M. (2017). Effects of surface modification on dispersion, mechanical, thermal and dynamic mechanical properties of injection molded PLA-hydroxyapatite composites. Composites Part A, Applied Science and Manufacturing, 103, 96-105.

Atak, B. H., Buyuk, B., Huysal, M., Isik, S., Senel, M., Metzger, W., ... Cetin, G. (2017). Preparation and characterization of amine functional nano-hydroxyapatite/chitosan bionanocomposite for bone tissue engineering applications. Carbohydrate Polymers, 164, 200-213.

Babick, F. (2016). Suspensions of colloidal particles and aggregates. Springer.

Burr, D. B., \& Allen, M. R. (2013). Basic and applied bone biology. Elsevier Science.

Carneiro-da-Cunha, M. G., Cerqueira, M. A., Souza, B. W., Teixeira, J. A., \& Vicente, A. A. (2011). Influence of concentration, ionic strength and $\mathrm{pH}$ on zeta potential and mean hydrodynamic diameter of edible polysaccharide solutions envisaged for multinanolayered films production. Carbohydrate Polymers, 85(3), 522-528.

Casarrubios, L., Matesanz, M. C., Sanchez-Salcedo, S., Arcos, D., Vallet-Regi, M., \& Portoles, M. T. (2016). Nanocrystallinity effects on osteoblast and osteoclast response to silicon substituted hydroxyapatite. Journal of Colloid and Interface Science, 482, $112-120$.

Chen, F., Wang, Z.-C., \& Lin, C.-J. (2002). Preparation and characterization of nano-sized hydroxyapatite particles and hydroxyapatite/chitosan nano-composite for use in biomedical materials. Materials Letters, 57(4), 858-861.

Chen, J., Zhang, G., Yang, S., Li, J., Jia, H., Fang, Z., ... Zhang, Q. (2011). Effects of in situ and physical mixing on mechanical and bioactive behaviors of nano hydroxyapatite-chitosan scaffolds. Journal of Biomaterials Science Polymer Edition, 22(15), 2097-2106.

Danilchenko, S. N., Kalinkevich, O. V., Pogorelov, M. V., Kalinkevich, A. N., Sklyar, A. M., Kalinichenko, T. G., ... Sikora, V. Z. (2011). Characterization and in vivo evaluation of chitosan-hydroxyapatite bone scaffolds made by one step coprecipitation method. Journal of Biomedical Materials Research Part A, 96(4), 639-647.

Fadeeva, I. V., Barinov, S. M., Fedotov, A. Y., \& Komlev, V. S. (2011). Interactions of calcium phosphates with chitosan. Doklady Chemistry, 441(2), 387-390.

Gomes, P. J., Silva, V. M., Quadros, P. A., Dias, M. M., \& Lopes, J. C. (2009). A highly reproducible continuous process for hydroxyapatite nanoparticles synthesis. Journal of Nanoscience and Nanotechnology, 9(6), 3387-3395.

Hu, Q., Li, B., Wang, M., \& Shen, J. (2004). Preparation and characterization of biodegradable chitosan/hydroxyapatite nanocomposite rods via in situ hybridization: A potential material as internal fixation of bone fracture. Biomaterials, 25(5), 779-785.

Jin, X., Zhuang, J., Zhang, Z., Guo, H., \& Tan, J. (2015). Hydrothermal synthesis of hydroxyapatite nanorods in the presence of sodium citrate and its aqueous colloidal stability evaluation in neutral pH. Journal of Colloid and Interface Science, 443, $125-130$.

Kickelbick, G. (2007). Introduction to hybrid materials. Hybrid materials: Synthesis, characterization, and applications. 1-46.

Kim, S.-H., Lim, B.-K., Sun, F., Koh, K., Ryu, S.-C., Kim, H.-S., ... Lee, J. (2009). Preparation of high flexible composite film of hydroxyapatite and chitosan. Polymer Bulletin, 62(1), 111.

Laranjeira, P. E., Martins, A. A., Lopes, J. C. B., \& Dias, M. M. (2009). NETmix ${ }^{\oplus}$, a new type of static mixer: Modeling, simulation, macromixing, and micromixing characterization. AIChE Journal, 55(9), 2226-2243.

Laranjeira, P. E., Martins, A. A., Nunes, M. I., Lopes, J. C. B., \& Dias, M. M. (2011). NETmix $^{\oplus}$, a new type of static mixer: Experimental characterization and model validation. AIChE Journal, 57(4), 1020-1032.

J. C. B. Lopes, P. E. L. Laranjeira, M. M. Q. Dias, A. A. Martins. (2005). Network Mixer and Related Mixing Process, PCT/IB2005/000647. European Patent EP172643 B1, US Patent 8434933 B2.

Nazeer, M. A., Yilgör, E., \& Yilgör, I. (2017). Intercalated chitosan/hydroxyapatite nanocomposites: Promising materials for bone tissue engineering applications. Carbohydrate Polymers, 175, 38-46.

Nguyen, V. S., Rouxel, D., Hadji, R., Vincent, B., \& Fort, Y. (2011). Effect of ultrasonication and dispersion stability on the cluster size of alumina nanoscale particles in aqueous solutions. Ultrasonics Sonochemistry, 18(1), 382-388.

Okada, T., Nobunaga, Y., Konishi, T., Yoshioka, T., Hayakawa, S., Lopes, M. A., Shirosaki, Y. (2017). Preparation of chitosan-hydroxyapatite composite mono-fiber using coagulation method and their mechanical properties. Carbohydrate Polymers, 175, 355-360.

Pangon, A., Saesoo, S., Saengkrit, N., Ruktanonchai, U., \& Intasanta, V. (2016). Hydroxyapatite-hybridized chitosan/chitin whisker bionanocomposite fibers for bone tissue engineering applications. Carbohydrate Polymers, 144, 419-427.

Peniche, C., Solís, Y., Davidenko, N., \& García, R. (2010). Materiales compuestos de quitosana e hidroxiapatita. Biotecnología Aplicada, 27(3), 202-210.

Pighinelli, L., \& Kucharska, M. (2013). Chitosan-hydroxyapatite composites. Carbohydrate Polymers, 93(1), 256-262.

Prakash, S., Mishra, R., Malviya, R., \& Sharma, P. K. (2014). Measurement techniques and pharmaceutical applications of zeta potential: A review.

Ruphuy, G., Saralegi, A., Lopes, J. C., Dias, M. M., \& Barreiro, M. F. (2016). Spray drying as a viable process to produce nano-hydroxyapatite/chitosan (n-HAp/CS) hybrid microparticles mimicking bone composition. Advanced Powder Technology, 27(2), 575-583.

Ruphuy, G., Souto-Lopes, M., Paiva, D., Costa, P., Rodrigues, A. E., Monteiro, F. J., Barreiro, M. F. (2018). Supercritical CO2 assisted process for the production of highpurity and sterile nano-hydroxyapatite/chitosan hybrid scaffolds. Journal of Biomedical Materials Research Part B, Applied Biomaterials, 106(3), 965-975.

Shakir, M., Jolly, R., Khan, A. A., Ahmed, S. S., Alam, S., Rauf, M. A., ... Farooqi, M. A. (2018). Resol based chitosan/nano-hydroxyapatite nanoensemble for effective bone tissue engineering. Carbohydrate Polymers, 179, 317-327.

Silva, V. M., Quadros, P. A., Laranjeira, P. E., Dias, M. M., \& Lopes, J. C. (2008). A novel continuous industrial process for producing hydroxyapatite nanoparticles. Journal of Dispersion Science and Technology, 29(4), 542-547.

Šupová, M. (2009). Problem of hydroxyapatite dispersion in polymer matrices: A review. Journal of Materials Science Materials in Medicine, 20(6), 1201-1213.

Šupová, M., Martynková, G. S., \& Barabaszová, K. (2011). Effect of nanofillers dispersion in polymer matrices: A review. Science of Advanced Materials, 3(1), 1-25.

Thein-Han, W., \& Misra, R. (2009). Biomimetic chitosan-nanohydroxyapatite composite scaffolds for bone tissue engineering. Acta Biomaterialia, 5(4), 1182-1197.

Uskoković, V., \& Uskoković, D. P. (2011). Nanosized hydroxyapatite and other calcium phosphates: Chemistry of formation and application as drug and gene delivery agents. Journal of Biomedical Materials Research Part B, Applied Biomaterials, 96B(1), $152-191$.

Venkatesan, J., \& Kim, S. K. (2010). Chitosan composites for bone tissue engineering-An overview. Marine Drugs, 8(8), 2252-2266.

Vucinic, D. R., Radulovic, D. S., \& Deusic, S. D. (2010). Electrokinetic properties of hydroxyapatite under flotation conditions. Journal of Colloid and Interface Science, 343(1), 239-245.

Wilson, O. C., Jr, \& Hull, J. R. (2008). Surface modification of nanophase hydroxyapatite with chitosan. Materials Science and Engineering C, 28(3), 434-437. 
Xianmiao, C., Yubao, L., Yi, Z., Li, Z., Jidong, L., \& Huanan, W. (2009). Properties and in vitro biological evaluation of nano-hydroxyapatite/chitosan membranes for bone guided regeneration. Materials Science and Engineering C, 29(1), 29-35.

Yamaguchi, I., Tokuchi, K., Fukuzaki, H., Koyama, Y., Takakuda, K., Monma, H., ... Tanaka, J. (2001). Preparation and microstructure analysis of chitosan/hydroxyapatite nanocomposites. Journal of Biomedical Materials Research Part A, 55(1), $20-27$.
Zhao, F., Yin, Y., Lu, W. W., Leong, J. C., Zhang, W., Zhang, J., ... Yao, K. (2002) Preparation and histological evaluation of biomimetic three-dimensional hydroxyapatite/chitosan-gelatin network composite scaffolds. Biomaterials, 23(15), $3227-3234$.

Zima, A. (2018). Hydroxyapatite-chitosan based bioactive hybrid biomaterials with improved mechanical strength. Spectrochimica Acta Part A, Molecular and Biomolecular Spectroscopy, 193, 175-184. 